\title{
Cancer Imaging Program
}

National Cancer Institute

\section{Source}

National Cancer Institute. Cancer Imaging Program. NCI Thesaurus. Code C49047.

The mission of the Cancer Imaging Program, National Cancer Institute, is to promote and support Cancer-related basic, translational and clinical research in imaging sciences and technology, and integration and application of these imaging discoveries and developments to the understanding of cancer biology and to the clinical management of cancer and cancer risk. 